SUBJECT AREAS:

MICROBIAL ECOLOGY

DNA METABOLISM

OBESITY

Received

22 October 2014

Accepted

9 February 2015

Published

30 March 2015

Correspondence and requests for materials should be addressed to

O.C. (o.carnevali@ univpm.it)

\section{* These authors contributed equally to} this work. \title{
lipid content by changing gut microbiota and host transcription of genes involved in lipid metabolism
}

\author{
Silvia Falcinelli ${ }^{1}$, Simona Picchietti ${ }^{2}$, Ana Rodiles ${ }^{3}$, Lina Cossignani ${ }^{4}$, Daniel L. Merrifield ${ }^{3 *}$, \\ Anna Rita Taddei ${ }^{5}$, Francesca Maradonna', Ike Olivotto', Giorgia Gioacchini' \& Oliana Carnevali'1*
}

'Dipartimento di Scienze della Vita e dell'Ambiente, Università Politecnica delle Marche, Ancona, Italy, ${ }^{2}$ Department for Innovation in Biological, Agro-food and Forest Systems (DIBAF), University of Tuscia, Viterbo, Italy, ${ }^{3}$ Aquatic Animal Nutrition and Health Research Group, School of Biological Sciences, Plymouth University, PL4 8AA, UK, ${ }^{4}$ Dipartimento di Scienze Economico-Estimative e degli Alimenti, Sezione di Chimica Bromatologica, Biochimica, Fisiologia e Nutrizione, Università degli Studi di Perugia, Perugia, Italy, ${ }^{5}$ Section of Electron Microscopy, Great Equipment Center. Tuscia University, 01100 Viterbo, Italy.

The microbiome plays an important role in lipid metabolism but how the introduction of probiotic communities affects host lipid metabolism is poorly understood. Using a multidisciplinary approach we addressed this knowledge gap using the zebrafish model by coupling high-throughput sequencing with biochemical, molecular and morphological analysis to evaluate the changes in the intestine. Analysis of bacterial $16 \mathrm{~S}$ libraries revealed that Lactobacillus rhamnosus was able to modulate the gut microbiome of zebrafish larvae, elevating the abundance of Firmicutes sequences and reducing the abundance of Actinobacteria. The gut microbiome changes modulated host lipid processing by inducing transcriptional down-regulation of genes involved in cholesterol and triglycerides metabolism (fit2, agpat4, dgat2, mgll, $h n f 4 \alpha$, scap, and cck) concomitantly decreasing total body cholesterol and triglyceride content and increasing fatty acid levels. $L$. rhamnosus treatment also increased microvilli and enterocyte lengths and decreased lipid droplet size in the intestinal epithelium. These changes resulted in elevated zebrafish larval growth. This integrated system investigation demonstrates probiotic modulation of the gut microbiome, highlights a novel gene network involved in lipid metabolism, provides an insight into how the microbiome regulates molecules involved in lipid metabolism, and reveals a new potential role for $L$. rhamnosus in the treatment of lipid disorders.

$\mathrm{P}$ robiotics positively modulate the gut microbiota, and recent studies revealed that host microbiota can modify host nutrient metabolism and energy balance both in germ free mice and zebrafish ${ }^{1-2}$. The gut microbiota has been considered as one of the most important factors influencing host metabolism, energy and lipid metabolism, fat distribution, insulin sensitivity and growth performance ${ }^{3}$.

Considering the increasing number of diseases associated with lipid metabolic disturbances, several studies have focused on the ability of probiotics, mainly Lactobacillus and Bifidobacterium, to decrease serum lipid content (e.g. cholesterol and triglycerides) $)^{1,3}$. Numerous hypotheses have been proposed for the mechanisms involved in the reduction of cholesterol by probiotics in the gut and their impact on the energy of the host ${ }^{4-5}$.

In general, the lipid metabolic pathway involves a considerable number of genes which regulate lipid synthesis, traffic, storage and homeostasis. Fish and mammals use triglycerides (TAG) and cholesterol derived from the diet as the main source of energy ${ }^{6}$. In eukaryotes, both the glycerol phosphate and the monoacylglycerol pathways represent the two main processes to resynthesize $\mathrm{TAG}^{7}$. TAG synthesis via the glycerol phosphate pathway mainly takes place in the liver and adipose tissues. ${ }^{8}$. Briefly, this process involves the acylation of glycerol 3phosphate by glycerol-3-phosphate acyltransferase (GAPT) and the conversion of diacylglycerol (DAG) into TAG by diacylglycerol acyltransferase (DGAT) ${ }^{8-10}$.

In order for the intestine to absorb TAG, TAG must first be hydrolyzed trough a three-step process catalyzed by adipose triglyceride lipase, hormonesensitive lipase, and monoacylglycerol lipase (MGLL) ${ }^{11-12}$. In particular, recent studies showed that MGLL is located in villi, enterocytes and adipocytes and is involved in the final step 
of TAG hydrolysis, specifically, hydrolyzing monoglycerides to free glycerol and fatty acids ${ }^{13-15}$. MGLL is also involved in the regulation 2 -arachidonoylglycerol signalling ${ }^{16}$. Free glycerol and fatty acids are then taken up by the enterocytes, resynthesized into new TAG and either incorporated into nascent chylomicrons for secretion or stored as lipid droplets (LDs) ${ }^{16-17}$

The monoacylglycerol pathway takes place in the intestine; the biosynthesis of TAG starts with the formation of bonds between monoacylglycerol and fatty acyl-CoA, catalyzed by monoacylglycerol acyltransferase, forming $\mathrm{DAG}^{18}$. Later, DGAT catalyzes a reaction that produces TAG from DAG and fatty acyl-CoA ${ }^{18}$. TAG together with sterol esters and phospholipids form LDs, which are cytosolic structures located in numerous cell types including enterocytes, which play a crucial role in the energy homeostasis in all eukaryotes ${ }^{19}$.

On the other hand, free cholesterol from biliary or dietary origin is absorbed by the enterocytes and incorporated into chylomicrons as cholesterol ester (CE), then transported into the lymphatic system ${ }^{6}$.

The present study aimed at evaluating the effect of supplementation of the probiotic L. rhamnosus on the gut microbial community and lipid metabolism of zebrafish (Danio rerio).

In order to achieve this, a wide network of genes involved in lipid metabolism were examined, and the effect of L. rhamnosus on intestinal epithelial structures, total body cholesterol, TAG and non-polar fatty acids and zebrafish larval growth was investigated.

\section{Results}

Probiotic modulation of the digestive tract microbiome. Highthroughput sequence analysis of bacterial 16S rRNA V3 region was conducted on samples at $6 \mathrm{dpf}$. The analysis revealed a highly diverse microbiota with a total of 200 thousand unique reads (with an average length of $166.0 \pm 6.5 \mathrm{bp}$ ), representing 218 OTUs, from over 1.2 million raw reads. Using default parameters, a de novo UCHIME algorithm ${ }^{20}$ was used to identify potential chimeric sequences, which accounted for $5.1 \%$ of the total sequences. The alpha rarefaction plot of observed species reached a saturation phase at approximately 169 OTUs indicating that adequate sequence coverage was obtained to reliably describe the full diversity present in the samples (Figure 1A). This was verified by the Good's coverage estimation values of $>99.9 \%$ (Table 1). The bacterial branch distance, which measures the complete phylogenetic diversity represented within a community $^{21}$, demonstrated a significantly lower microbial diversity in the probiotic group $(2.34 \pm 0.14)$ compared to control group $(2.65$ $\pm 0.16)(P<0.05)$ (Table 1). In order to evaluate relationships among samples based on differences in phylogenetic diversity, two-dimensional principal coordinate analysis (PCoA) plots were calculated from weighted UniFrac distances for the evaluation of the community composition $^{22}$ (Figure 1D) and Bray-Curtis metrics were used for the estimation of the dissimilarity among samples ${ }^{23}$ (Figure 1E). Both PCoA plots show clustering of the replicates from the probiotic treatment away from the control samples, suggesting that the probiotic modified the bacterial communities in a characteristic direction.

In general, the bacterial communities in both treatments were dominated by three phyla: Firmicutes, Proteobacteria and Actinobacteria. The distinguishable separation of bacterial communities found in PCoA was accompanied with differences in the composition of the gut microbiota. A small proportion of the reads were identified as belonging to the Bacteriodetes (4.3\%), Fusobacteria (2.0\%), and Planctomycetes $(1.6 \%)$ phyla in the control, which were considerably less abundant in the probiotic group (where Bacteriodetes and Fusobacteria were not detected and Planctomycetes $=0.3 \%$ of the reads) (Figure $1 \mathrm{~B}$ ), and higher relative abundance of Firmicutes was observed in the probiotic group $(P<0.05$, Figure 1B). Most OTUs were resolved to class-level, others by order and some to genus level (Figure 1B-C). Firmicutes were dominated by the class Bacilli, with this class more abundant in the treated group than the control $(P<0.05)$.
Lactobacillus spp. were significantly more abundant in the probiotic treated larvae (accounting for 33.0\% of the total reads) than the control larvae (accounting for $8.1 \%$ of the reads) $(P<0.05)$, BLAST results identified the dominant lactobacilli as L. rhamnosus (NR-102778) in the probiotic treated samples, accounting for $100 \%$ of the lactobacilli reads, whereas lactobacilli reads from the control comprised Lactobacillus reuteri and L. rhamnosus. The abundance of reads assigned to the Streptococcus genera did not differ significantly between the groups, but BLAST revealed that the species composition of this genera differed between the treatment groups: Streptococcus thermophilus (NR- 074827) dominated the probiotic samples and the control samples were dominated by an uncultured Streptococcus sp. most closely related to Streptococcus sanguinis ( $94 \%$ alignment similarity). A core microbiome of OTUs, detected in both treatment groups, was identified and comprised 27 OTUs (Figure 1F); these were the Streptococcus, Pseudomonas, Lactobacillus, Acidovorax, Actinomyces, Enhydrobacter, Ochrobactrum, Phenylobacterium, Acinetobacter, Escherichia, Aminobacter, Corynebacterium and Agrobacterium genera and 14 additional OTUs which could not be accurately identified at the genus level. Five OTUs were unique to the fish treated with the probiotic (Janthinobacterium, Gluconacetobacter, Faecalibacterium and two unidentified OTUs) and the 11 OTUs were uniquely detected in the control fish (Chryseobacterium, Sneathia, Mycobacterium, Kocuria, Hymenobacter, Finegoldia and five unidentified OTUs) (Figure 1F).

L. rhamnosus affects the expression of genes involved in lipid metabolism. The high-throughput sequencing results evidenced a change in gut microbiota community exposed to L. rhmanosus. In order to gain further insight, we next wanted to evaluate effect of microbiota modulation on lipid metabolism, and thus the expression of genes related with lipid pathways were assessed.

At hatching, when probiotic administration was not yet supplied, the gene expression levels of agpat4, a gene encoding for an enzyme involved in the conversion of lysophosphatidate to phosphatidate in the glycerol phosphate pathway ${ }^{24}$, was almost undetectable (Figure 2A). During larval development of the control fish this gene reached the highest level of expression at $96 \mathrm{hpf}$ and significantly decreased at 6 and $8 \mathrm{dpf}$ (the last stage analyzed in the present study). A similar trend was recorded in L. rhamnosus treated fish with the highest levels of agpat4 gene expression detected at $96 \mathrm{hpf}$ and significantly lower levels at 6 and $8 \mathrm{dpf}$. Comparison between the two experimental groups revealed a significant down-regulation of agpat4 in the probiotic treated larvae with respect to the control group, in all the developmental stages analyzed (96 hpf, $6 \mathrm{dpf}$ and $8 \mathrm{dpf}$ ).

The gene expression analysis of dgat2, a gene encoding for a protein that catalyzes a reaction which produces TAG from DAG and fatty acyl-CoA ${ }^{8}$, was clearly detectable at hatching (Figure $2 \mathrm{~B}$ ); at $96 \mathrm{hpf}$ a significant decrease occurred and a successive significant increase at $6 \mathrm{dpf}$ and $8 \mathrm{dpf}$ was observed. In treated larvae, the lowest dgat 2 level was detected at $96 \mathrm{hpf}$, gradually increasing at $6 \mathrm{dpf}$ and reaching the highest level at $8 \mathrm{dpf}$. The comparison between control and treated groups evidenced similar levels at $96 \mathrm{hpf}$, while significantly lower levels were observed in the probiotic treated group at 6 and $8 \mathrm{dpf}$.

The analysis of $m g l l$ expression levels, which encodes for a serine hydrolase that preferentially hydrolyses monoacylglycerols [e.g. 2arachidonoylglycerol; (2-AG)] to free fatty acid and glycerol ${ }^{14}$, evidenced its presence at hatching (Figure 2C). In the control, the expression was significantly higher at $96 \mathrm{hpf}$, significantly decreased at $6 \mathrm{dpf}$ and a successively significant increase occurred at $8 \mathrm{dpf}$. The same trend was observed in treated larvae at all the developmental stages analyzed. The comparison between the two experimental groups revealed that probiotic treatment induced significantly higher expression at $96 \mathrm{hpf}$ and $6 \mathrm{dpf}$ while no difference was apparent at $8 \mathrm{dpf}$. 
(A)

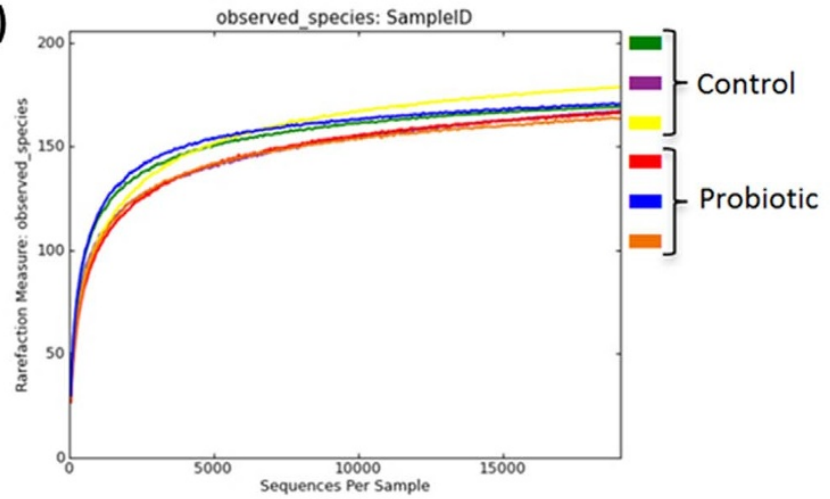

(C)

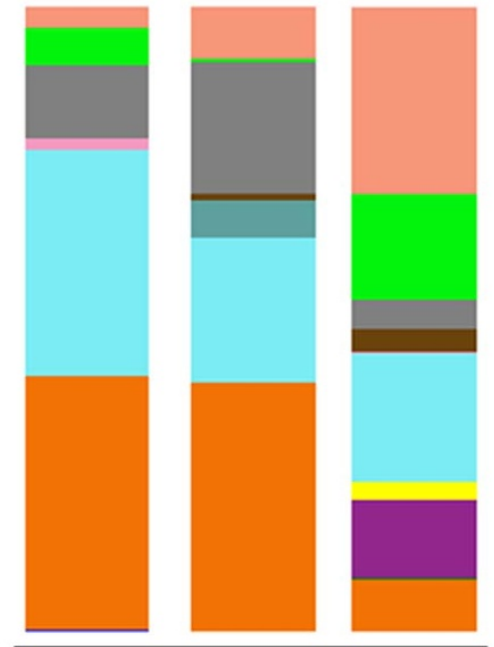

Control

(D)
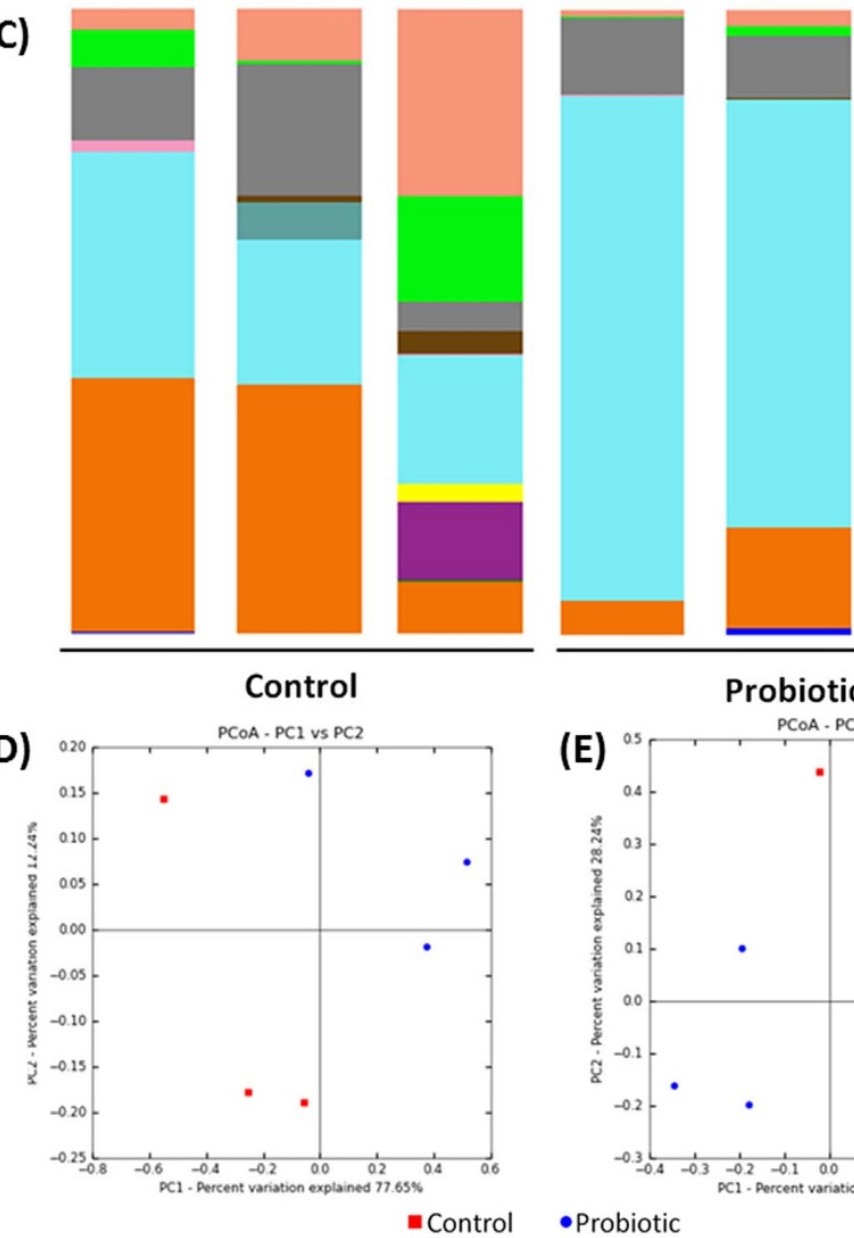

(E)
(B)
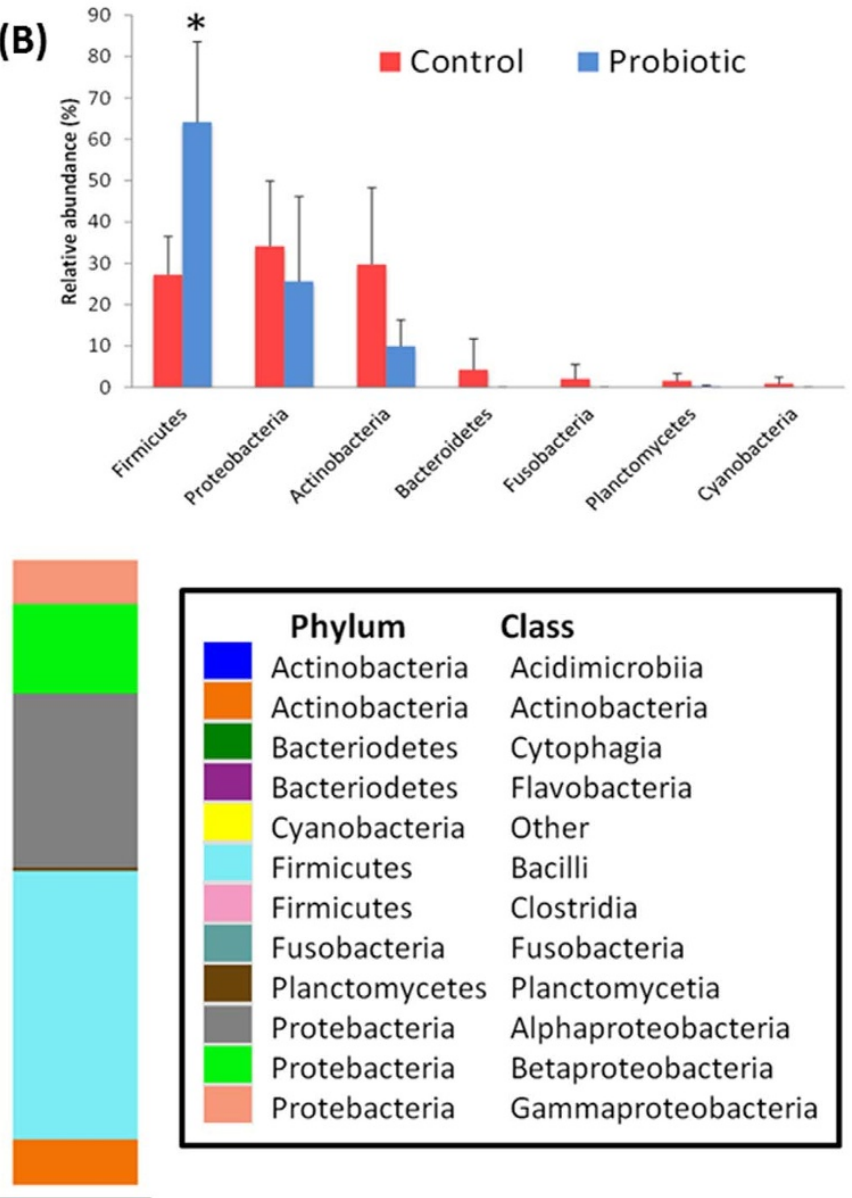

\begin{tabular}{|ll|}
\hline Phylum & Class \\
Actinobacteria & Acidimicrobiia \\
Actinobacteria & Actinobacteria \\
Bacteriodetes & Cytophagia \\
Bacteriodetes & Flavobacteria \\
Cyanobacteria & Other \\
Firmicutes & Bacilli \\
Firmicutes & Clostridia \\
Fusobacteria & Fusobacteria \\
Planctomycetes & Planctomycetia \\
Protebacteria & Alphaproteobacteria \\
Protebacteria & Betaproteobacteria \\
Protebacteria & Gammaproteobacteria \\
\hline
\end{tabular}

\section{Probiotic}

PCOA. PC1 vS PC2

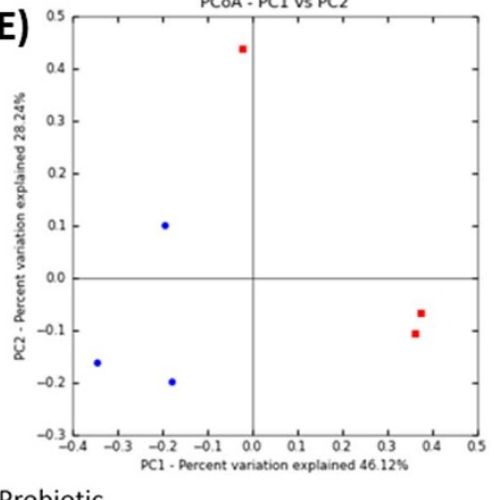

(F)

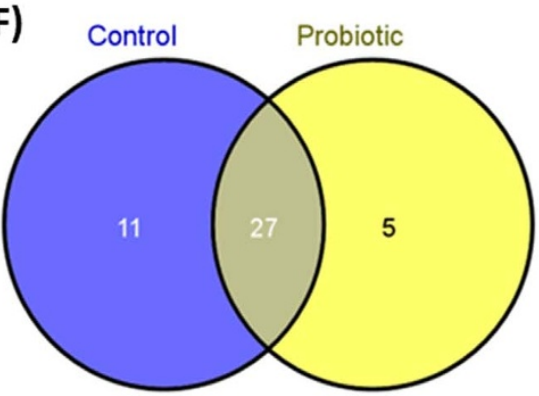

Figure 1 Digestive tract bacterial community analysis of 6 dpf zebrafish larvae. (A) Alpha rarefaction plot of observed species. (B) Relative abundance of reads assigned to phyla. (C) Stacked bar chart representing the relative abundance of bacterial phylum and classes. (D) and (E) Principal coordinates analysis (PCoA) plots using Bray-Curtis metric and weighted Unicfrac distances, respectively. (F) Unique and shared OTUs (at the level of genera) present in the treatment groups.

The analysis of $h n f 4 \alpha$, a gene encoding for a protein implicated in the metabolism of cholesterol, fatty acids and amino acids ${ }^{25}$, evidenced its presence at hatching, and in both control and probiotic treated groups peaked at $96 \mathrm{hpf}$, and subsequently significantly decreased at $6 \mathrm{dpf}$ reaching the lowest level at $8 \mathrm{dpf}$ (Figure 2D). Compared to the control, $h n f 4 \alpha$ gene expression was down regulated by the probiotic at $6 \mathrm{dpf}$.

The fit 2 gene, encoding for a protein involved in fat storage ${ }^{19}$, transcript analysis in the control group showed the highest level at hatching; similar levels were maintained at $96 \mathrm{hpf}$ followed by a significant decrease at 6 and $8 \mathrm{dpf}$ (Figure 2E). In treated larvae, higher levels were observed at $96 \mathrm{hpf}$ and $8 \mathrm{dpf}$ while at $6 \mathrm{dpf}$ the lowest levels were detected. Comparing the levels of $f$ it 2 gene between the treatments, lower levels were observed at $96 \mathrm{hpf}$ and $6 \mathrm{dpf}$ in the probiotic treatment.

The analysis of the expression of scap (Figure 2F), encoding for Cleavage Activation Protein which activates SREBPs which regulate the transcription of genes involved in uptake and synthesis of de novo cholesterol and fatty acids ${ }^{26}$, evidenced highest levels at hatching. In both experimental groups a significant decrease of gene expression 
Table 1 | Alpha diversity metrics of observed species, phylogenetic diversity (PD), Chaol, Shannon's diversity and Good's coverage of zebrafish larvae of $6 \mathrm{dpf}$ (mean \pm s.d.; $n=3$ )

\begin{tabular}{lccccc} 
& Observed species & Chaol & Shannon & Phylogenetic distance & Good's coverage \\
\hline Control & $195.1 \pm 9.2$ & $209.3 \pm 12.2$ & $5.57 \pm 0.37$ & $2.74 \pm 0.14^{\mathrm{b}}$ & $0.9992 \pm 0.0002$ \\
Probiotic & $194.5 \pm 1.5$ & $206.5 \pm 1.1$ & $5.92 \pm 0.14$ & $2.45 \pm 0.05^{\mathrm{a}}$ & $0.9994 \pm 0.0001$ \\
\hline
\end{tabular}

Values with different superscripts, within the same column, are significantly different at $P<0.05$.

was detected at all stages analysed compared to the levels at hatching. No significant differences were observed between the control and probiotic groups at any of the developmental stages analyzed.

Finally the analysis of $c c k$, which encodes for a peptide hormone responsible for gallbladder contraction and pancreatic enzyme secretion $^{27}$, was clearly detectable at hatching (Figure $2 \mathrm{G}$ ); at $96 \mathrm{hpf}$ a significant decrease was detected and a successive significant increase at $6 \mathrm{dpf}$ was observed in the control group compared to the time of hatching. Finally, at $8 \mathrm{dpf}$, the gene expression level reached the same level of that at the time of hatching. The same trend was observed in treated group. The comparison between control and treated groups evidenced significantly higher levels in the probiotic treated group in all the developmental stages analyzed (96 hpf, $6 \mathrm{dpf}$ and $8 \mathrm{dpf}$ ).

Modulation of microbiota decreased the total body cholesterol and triglyceride levels. Triglycerides and cholesterol derived from the diet are used from both fish and mammals as the main source of energy which can be released rapidly on demand ${ }^{6}$. TAGs represent the major source of lipid storage and cholesterol and are an important component of cell membranes; they are also the precursor molecules for the synthesis of steroid hormones, bile salts and vitamin $\mathrm{D}^{28-29}$. However, it has been reported that excess TAG storage as well as high serum cholesterol levels cause several metabolic disorders including Type 2 diabetes, hypertension and atherosclerosis ${ }^{30}$. To gain further insight into the probiotic effects on lipid metabolism, the zebrafish total cholesterol and TAG content were evaluated. The High Performance Liquid Chromatography (HPLC) analyses performed on total body samples evidenced that zebrafish treated with L. rhamnosus had significantly lower levels of total cholesterol $(0.083 \pm 0.006 \mu \mathrm{g} /$ $\mathrm{mg})$ and TAG $(0.040 \pm 0.003 \mu \mathrm{g} / \mathrm{mg})$ compared to the total cholesterol $(0.131 \pm 0.014 \mu \mathrm{g} / \mathrm{mg})$ and TAG $(0.088 \pm 0.010 \mu \mathrm{g} /$ $\mathrm{mg})$ levels in the control group $(P<0.05)$ (Table 2$)$.

Ultrastructural analysis by Transmission Electron Microscopy (TEM) revealed longer enterocytes and microvilli heights in the intestine of probiotic treated zebrafish. At $6 \mathrm{dpf}$ the alimentary canal of zebrafish comprises the mouth, pharynx, esophagus, intestinal bulb, mid-intestine, posterior intestine and anal opening. The yolk is completely resorbed ${ }^{31}$. In the intestinal bulb, the rostral region of the gut, the lumen was greatly expanded and the epithelial layer was arranged into broad, irregular folds. The intestine of control group had an undamaged epithelial barrier and no signs of degradation (Figure 3A). The intestines exposed to the probiotic $L$. rhamnosus had an intact epithelial barrier, lack of cell debris in the lumen and no signs of damage (Figure 3B). At this stage the intestine consisted mainly of uniform, columnar, polarized epithelia, with an apical brush border, lateral cell border, and basal basement membrane. Both in control and treated groups (Figure 3C-D), columnar-shaped enterocytes were joined apically through a complex set of junctional complexes (tight junctions, adherens junctions and desmosomes) that restrict the movement of membrane components between apical and basolateral cellular domains, and serve as a barrier to the paracellular space through which luminal contents may otherwise enter the organism (Figure 3E). In the probiotic treated larvae the enterocytes lengths were significantly higher $(42.24 \pm 1.78 \mu \mathrm{m})$ compared to the control $(34.64 \pm 2.06 \mu \mathrm{m})$ and significantly longer microvilli on the apical surface were observed in L. rhamnosus exposed larvae $(1.21 \pm$ $0.09 \mu \mathrm{m})$ with respect to the control $(1.06 \pm 0.08 \mu \mathrm{m})(P<0.05)$ (Figure 3F-G). The enterocyte's apical cytoplasm contained copious spherical mitochondria and endoplasmic reticulum. In addition, lipid accumulations were present in the apical and basal cytoplasm of both control and treated groups (Figure $3 \mathrm{C}-\mathrm{D} ; \mathrm{H}-\mathrm{I}$ ). These accumulations were determined to be lipid droplets by their uniform interior, association with endoplasmic reticulum and mitochondria, and presence of a bounding monolayer membrane. In probiotic treated larvae the diameter of the lipid droplets $(2.37 \pm$ $0.37 \mu \mathrm{m})$ was significantly smaller compared to the control $(4.10 \pm$ $0.40 \mu \mathrm{m})(P<0.05)$. Supplemental material: see also supplemental Figure S3 (A) for enterocyte lengths (related to Figure 3C-D), Figure S3 (B) for lipid droplet diameter (related to Figure 3 C-D; H-I), and Figure S3 (C) for microvilli lengths (related to Figure $3 \mathrm{~F}-\mathrm{G}$ ).

In vivo non-polar fatty acids localization with BODIPY 505/515 staining revealed accumulations in the gallbladder and intestine of probiotic treated fish. Teleosts and mammals have complex digestive physiologies but share a high degree of anatomical similarity ${ }^{32}$. Zebrafish, like humans, use TAG as a major source of lipid, and in order for the intestine to absorb TAG, processing by digestive enzymes and bile is require ${ }^{33}$. Bile and enzymes pass from the gallbladder and arrive to the intestinal lumen where TAG are emulsified and form micelles and finally, free fatty acids (FFAs) and monoacylglycerols are taken up by intestine ${ }^{34}$. It has been demonstrated that FFAs have an important role in gallbladder secretion $^{35}$. We used BODIPY 505/515 (borondipyrromethene fluorescent moiety) staining as a non-invasive in vivo method in order to visualize the dynamics of non-polar fatty acids in living tissues by confocal microscopy. Imaging revealed similar green fluorescence intensity in the intestine of both control $(320 \pm 34$ a.u.) (Figure $4 \mathrm{~A}-\mathrm{B})$ and probiotic treated zebrafish larvae (338 \pm 52 a.u.) at $6 \mathrm{dpf}$ (Figure $4 \mathrm{C}-\mathrm{D})(P<0.05)$. On the contrary, probiotic treated larvae exhibited significantly higher fluorescent signal in the gallbladder $(535 \pm 66$ a.u.) with respect to the control $(340 \pm 53$ a.u. $)(P<0.05)$ highlighting an accumulation of non polar-fatty acids.

Supplemental figure: Figure S4 (E) shows graph with statistical difference of intestinal fluorescence of both control and treated larvae (Figure $4 \mathrm{~A}-\mathrm{B}-\mathrm{C}-\mathrm{D})$.

Treatment with $L$. rhamnosus improves the growth of zebrafish larvae. Since we reported compelling evidence of gut community modulation and in turn lipid metabolism modulation after administration of L. rhamnosus, by measuring total larval length and wet weight, we sought to determine if these changes impacted the growth of zebrafish larvae. At $96 \mathrm{hpf}$ no significance differences were observed in terms of length and weight in both control (length $3.3 \pm 0.17 \mathrm{~mm}$; weight $0.43 \pm 0.07 \mathrm{mg}$ ) and treated (length $3.4 \pm$ $0.11 \mathrm{~mm}$, weight $0.4 \pm 0.08 \mathrm{mg})$ larvae $(P>0.05)$. At $6 \mathrm{dpf}$ no significant difference of larval length was observed between control larvae $(3.5 \pm 0.16 \mathrm{~mm})$ and larvae exposed to L. rhamnosus (3.6 \pm $0.18 \mathrm{~mm})(P>0.05)$. Despite this, a significant increase of wet 


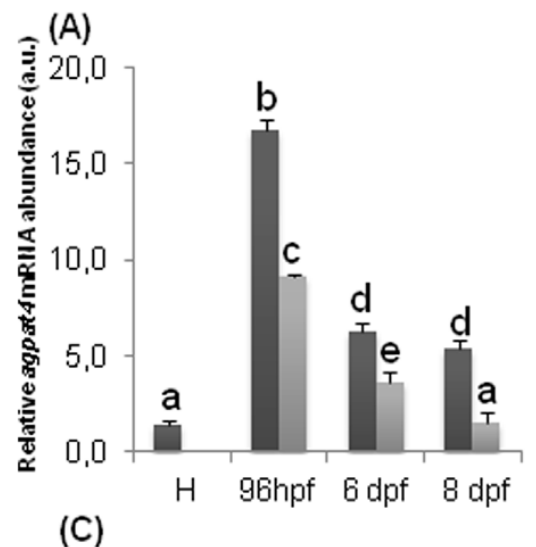

(B)

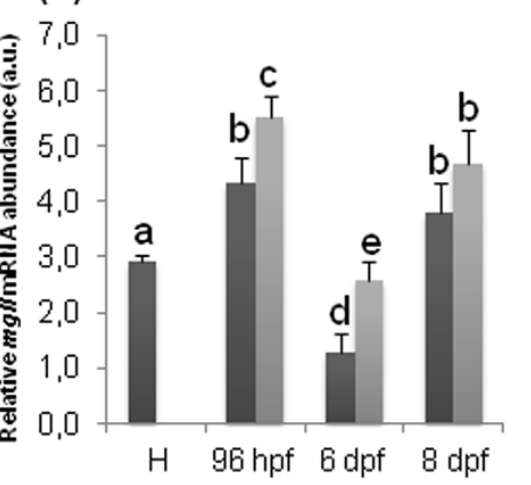

(E)
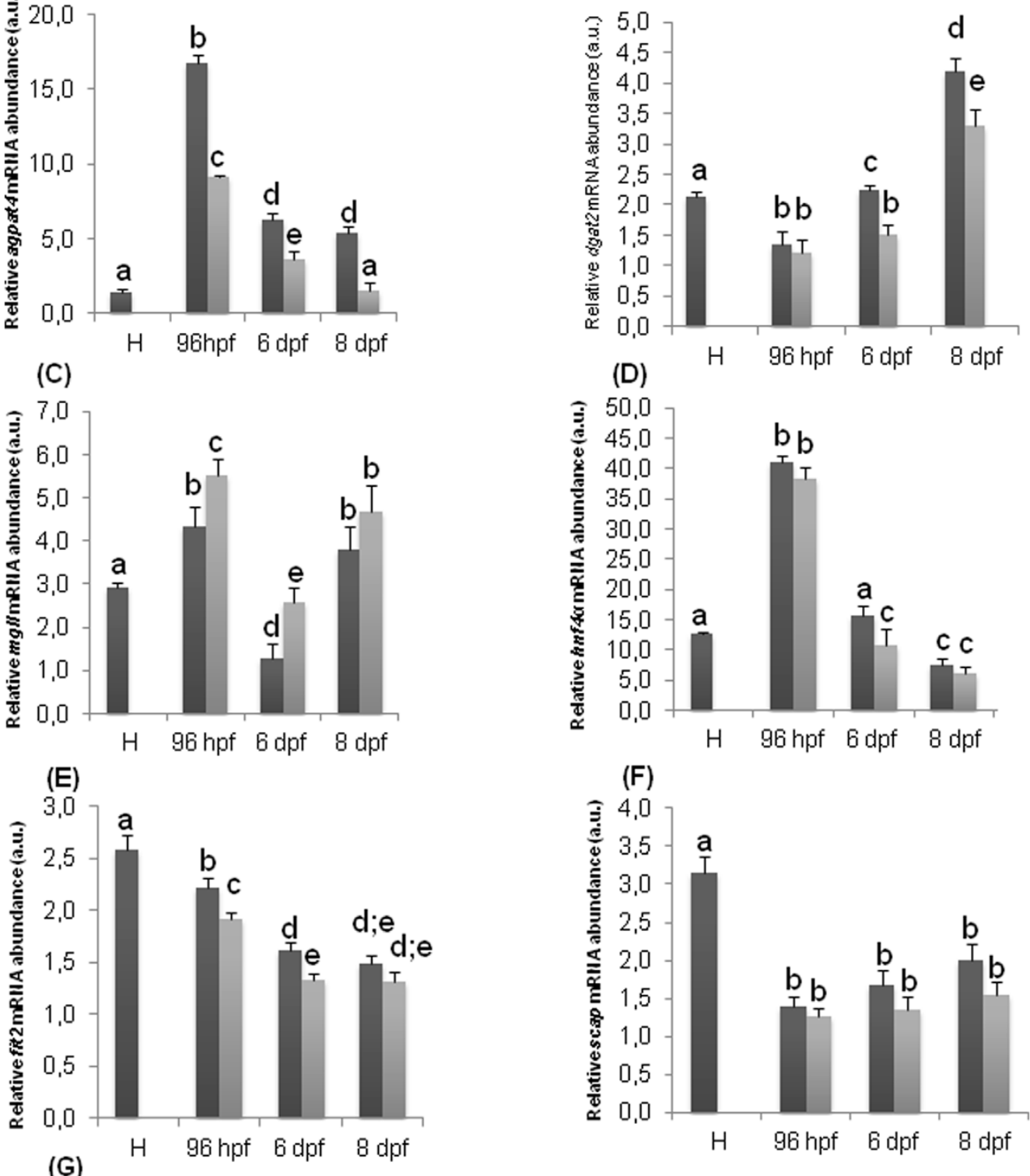

$(\mathrm{F})$
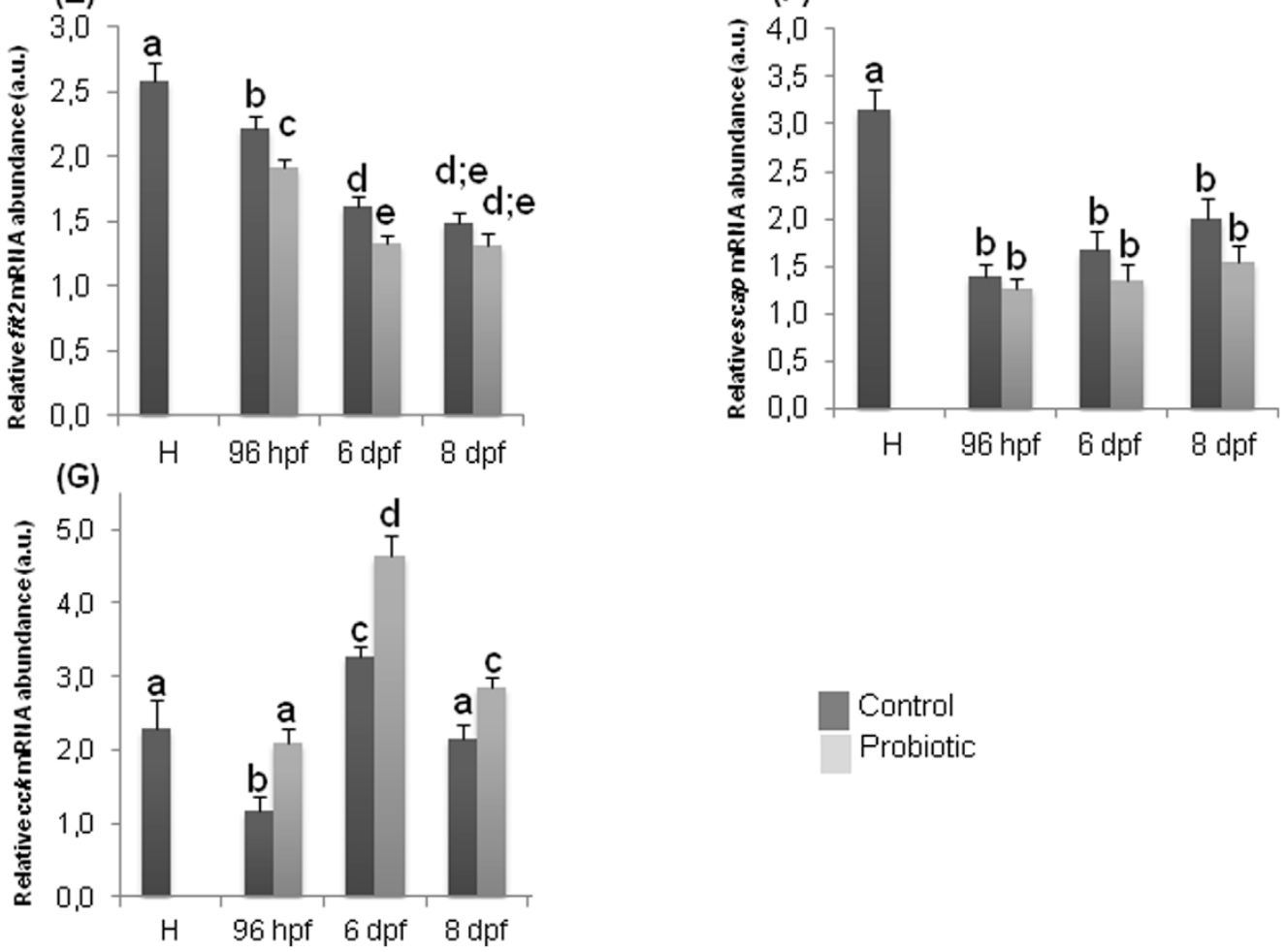

Control

Probiotic

Figure $2 \mid$ L. rhamnosus treatment modulates expression of genes involved in lipid metabolism. qRT-PCR analysis. Relative agpat (A), dgat2 (B), mgll (C), $h n f 4 \alpha(\mathrm{D})$, fit2 (E), scap (F), cck (G) gene expression normalized against $\beta$-act and arp, in pools of 15 zebrafish larvae from control and probiotic groups collected at hatching $(\mathrm{H}), 96 \mathrm{hpf}, 6 \mathrm{dpf}$ and $8 \mathrm{dpf}$. Assays were performed in triplicate. Values with different superscript letters are significantly different $(P<0.05)$.

weight was observed at $6 \mathrm{dpf}$ in the probiotic supplemented group $(1.19 \pm 0.09 \mathrm{mg})$ compared to the control group $(0.9 \pm 0.1 \mathrm{mg})$. At $8 \mathrm{dpf}$ a significant increase of total length $(3.9 \pm 0.12 \mathrm{~mm})$ concomitant with a significant increase of wet weight $(1.37 \pm$ $0.13 \mathrm{mg}$ ) was apparent in zebrafish larvae treated with the probiotic compared to the length $(3.6 \pm 0.21 \mathrm{~mm})$ and wet weight 
Table 2 | L. rhamnosus administration affects whole larval cholesterol and triglycerides content

\begin{tabular}{lccc} 
Parameter & \multicolumn{2}{c}{ Control } & \multicolumn{1}{c}{ Probiotic } \\
\cline { 2 - 3 } & Value & Value & $0.083 \pm 0.006^{*}$ \\
\hline Cholesterol $(\mu \mathbf{g} / \mu \mathbf{g}$ DNA) & $0.131 \pm 0.014$ & $0.040 \pm 0.003^{*}$ & 0.04 \\
TAG $(\mu \mathbf{g} / \mu \mathbf{g}$ DNA) & $0.088 \pm 0.010$ & 0.02 \\
\hline Prolue
\end{tabular}

Probiotics significantly decrease the content of cholesterol and triglyceride (TAG) in 6 dpf zebrafish larvae (pools 300 mg of larvae). Data are the mean \pm s.d. Data have been normalized with DNA content. Values with asterisk are significantly different $(P<0.05)$.

$(1.07 \pm 0.08 \mathrm{mg})$ detected in control group $(P<0.05)$ (Figure 5A$B)$. In all stages examined the viable larvae observed in both control and treated groups were $95-98 \%$.

\section{Discussion}

In the current study, the administration of L. rhamnosus induced changes to the microbial composition of the zebrafish digestive tract. The probiotic treatment reduced the presence of some genera which contain potential pathogens (e.g. Mycobacterium) and enhanced the abundance of reads assigned to the class Bacilli. Reads from the Lactobacillus genera were significantly elevated by the probiotic larvae and in addition the probiotic enhanced the presence of another lactic acid bacteria, Streptococcus thermophilus. This finding is in agreement with the previous study on 6-month old zebrafish fed dietary L. rhamnosus which demonstrated that the probiotic populated the intestinal tract and elevated the abundance Streptococcus thermophilus ${ }^{36}$. Streptococcus thermophilus and lactobacilli (e.g. Lactobacillus delbrueckii subsp. bulgaricus) are known symbionts as observed from their interactions as starter cultures in dairy products; Streptococcus thermophilus is suggested to provide folic acid and formic acid to the lactobacilli which in turn provides a source of amino acids ${ }^{37}$. The present study, and that of Gioacchini et al. ${ }^{36}$, would suggest that a similar symbiotic relationship may occur between L. rhamnosus and S. thermophilus in the digestive tract of zebrafish. Indeed, some strains of lactobacilli and streptococci have been reported to have symbiotic effects in mammals, subsequently reducing hepatic cholesterol levels in rats fed a fat- and cholesterolenriched diet ${ }^{38}$.

The in vivo mechanisms of the gut microbiota which influence host dietary lipid metabolism are still unknown, however Semova et al. ${ }^{1}$ identified that colonization of the zebrafish gut with Firmicutes promotes epithelial absorption and is involved in host energy balance of zebrafish larvae. Further studies are required to enhance our understanding of the roles that gut microbes play in lipid metabolism and specific studies should elucidate the extent to which the observed effects in the present study are L. rhamnosus-specific; for example, it is not apparent from the present study if the host effects could be driven by overall changes, elevations in microbial load or whether other probiotic species are capable of driving these effects.

Microvilli and enterocyte height are directly correlated with the gut function and health of the host; an increase of microvilli and enterocytes heights provide increased absorptive surface area. Our TEM analysis highlighted increased microvilli and enterocyte heights, suggesting that L. rhamnosus is able to expand intestine structures. These findings are in agreement with previous probiotic studies ${ }^{39-40}$ and are likely to contribute towards the improved growth performance of the zebrafish larvae in the present investigation. Additionally, it has been observed that gut microbiota can ameliorate the absorption of nutrients and minerals, in particular calcium ${ }^{41}$. In the intestine, microbial production of SCFAs, which may have been elevated by the probiotics, can decrease the $\mathrm{pH}$ which indirectly increases the solubility of minerals and subsequently their absorption $^{42}$. Furthermore, the symbiotic relationship between L. rhamnosus and $S$. thermophilus and their ability to generate metabolites, such as SCFAs, which can allow the host, especially the enterocytes, to use energy from the diet in a more efficent way. Is well known that enterocytes obtain a large proportion of their energy from the oxidation of luminally derived SCFAs and absorbed SCFAs not utilised by enterocytes may also provide additional energy at the systemic level for other cell types ${ }^{43}$. These events could positively affect the host, leading to an increase in host growth ${ }^{44}$.

Further, possible modulation of the expression of genes involved in lipid metabolism may also have contributed towards improved growth.

L. rhamnosus administration and the relative modulation of the gut microbiota was able to induce transcriptional changes to a suite of genes involved in the synthesis, transport, storage and homeostasis of lipids, in particular, cholesterol and TAG. TAG biosynthesis is catalysed by AGPAT4 and DGAT2 enzymes and recent studies in mice and rats showed that their inhibition decreases TAG synthesis ${ }^{45-47}$. In this study, agpat4 and dgat 2 transcription were both decreased by L. rhamnosus. Furthermore these transcriptional changes induced by L. rhamnosus administration reflected a significant reduction in TAG content observed in treated larvae. TAG, together with sterol esters and phospholipids, form LD and recent studies on mouse liver and muscle evidenced that knockdown of fit2 significantly decreases LD accumulation ${ }^{48}$. Recently, it was demonstrated that fit2 expression is ubiquitous in all zebrafish tissues ${ }^{49}$. In the present study, the lower level of fit 2 mRNA found in probiotic treated larvae was concomitant with the reduction in size of LD in the intestine of treated larvae, evidenced by TEM analyses. This result is consistent with a study showing that fit 2 knockdown in zebrafish resulted in a decrease of LD in the liver and intestine ${ }^{49}$.

Recently, Guo and co-workers ${ }^{50}$ demonstrated that stimulation of lipolysis may break down large LDs into smaller LDs, in order to increase the surface area available for lipases, which are able to hydrolyze TAG. We observed a reduction in triglyceride content and a decrease of LDs accumulation, suggesting that L. rhamnosus affects the expression of TAG metabolism related genes (agpat4, dgat2 and fit2) and may stimulate lipolysis. Our findings on LDs accumulation are also consistent with a previous study in Solea senegalensis which showed the ability of a probiotic from the Alteromonadaceae family to inhibit intestinal LDs accumulation ${ }^{51}$. Interestingly, BODIPY 505/515 staining evidenced an intense fluorescent signal in the gallbladder of probiotic treated larvae, indicating that this non-polar lipid can be absorbed by the intestine, successively taken up by the liver and secreted into the gallbladder, through hepatobiliary transport.

The enhancement of fluorescence in the gallbladder prompted us to speculate an accumulation of non-polar fatty acids and/or other hydrophobic region that constitute most of the lipids. The bile in the gallbladder is composed by several molecules and among these, bile acids and bile alcohol, composed by both hydrophobic and hydrophilic regions, are the most abundant molecules in the bile of vertebrates $^{52}$. Zebrafish bile contains bile alcohol ${ }^{52}$. The synthesis of bile alcohol is not thought to be influenced by diet, rather mostly regulated by the gut microbiota, which possess unique enzymes involved in deconjugation, oxidation and esterification of bile alcohol ${ }^{53}$; thus, this result could indicate an increase of non-polar lipid bile fraction 

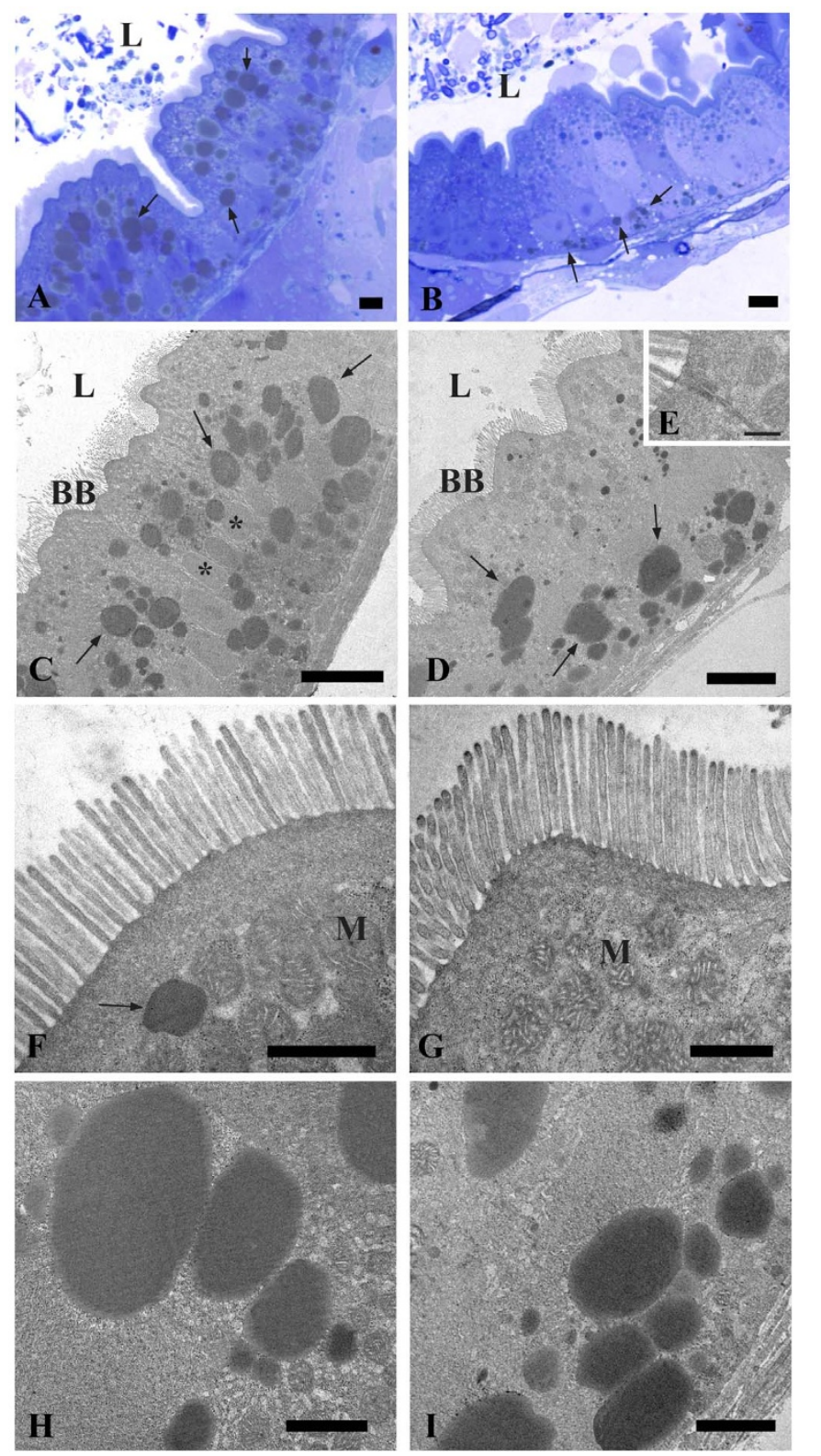

Figure 3 Transmission Electron Microscopy (TEM) shows the ultrastructure of the intestine in control and probiotic treated zebrafish. Thin section $(80 \mathrm{~nm})$ of $6 \mathrm{dpf}$ zebrafish showing the epithelial layer arranged into broad, irregular folds (A). Intact epithelial barrier, lack of cell debris in the lumen and no signs of damage in larvae exposed to probiotic L. rhamnosus (B). Electron micrographs showing uniform, columnar, polarized epithelia, with an apical brush border in control (C) and probiotic treated groups (D). Higher magnification of junctional complexes (E). Microvilli on the apical surface and copious spherical mitochondria in the enterocyte cytoplasm of control (F) and treated larvae (G). Higher magnification of the lipid droplets in the enterocytes of control larvae $(\mathrm{H})$ and in the probiotic treated intestine (I). BB: brush border; L: lumen; M: mitochondria; *: nucleus; arrows: lipid droplets. Scale bar: $5 \mu \mathrm{m}$ in (A, B); $10 \mu \mathrm{m}$ in (C, D); $500 \mathrm{~nm}$ in (E), $1 \mu \mathrm{m}$ in (F, G); $2 \mu \mathrm{m}$ in $(\mathrm{H}, \mathrm{I})$. (See also Figure $\mathrm{S} 3$ ).

production as a result of the probiotic activity, as previously demonstrated by Pavlović et al. ${ }^{54}$

It has been evidenced that free fatty acids stimulate CCK, which induces the contraction of the gallbladder to release bile into the intestine and solubilize lipids into micelles that are hydrolized by pancreatic lipase ${ }^{35}$. Our results demonstrate an up-regulation of cck gene expression levels concomitant with a higher content of non-polar fatty acids in the gallbladder after the probiotic supplementation. This could indicate increased bile production and hepatobiliary function, reduced bile secretion into the intestine, altered intestinal motility rates, improved the enterohepatic cycling of bile acids and/or bile transportation ${ }^{55-56}$.

Recently, MGLL has been described as one of the lipases involved in the final step of TAG hydrolysis ${ }^{57}$. Our results showed an upregulation of $m g l l$ gene expression level that reflected the lower TAG content observed, consistent with the presence of non-polar fatty acids (as a probable result of the breakdown of triglycerides) in the gallbladder after probiotic supplementation, which indicates the role of the digestive tract microbiota in the modulation of non-polar fatty acids metabolism.

In addition, recent studies showed that $h n f 4 \alpha$ knock-down reduces cholesterol content ${ }^{58-59}$. Our data indicate that L. rhamnosus treatment decreases the expression of the $h n f 4 \alpha$ gene, and therefore these results suggest that the modulation of gut microbiota, by inducing transcriptional changes, modulates cholesterol metabolism. The down-regulation of $h n f 4 \alpha$ after probiotic supplementation reflected a lower cholesterol content in the larvae. These findings are in agreement with a study which demonstrated cholesterol-lowering action by probiotics in mammals ${ }^{60}$. Cholesterol homeostasis is controlled by scap which plays an essential role in maintaining and controlling de novo synthesis of cholesterol, and therefore, is responsible for endogenous biosynthesis ${ }^{61}$. The maintenance of scap levels in the current study, concomitant with the decrease of cholesterol in treated larvae, suggests that the cholesterol reduction is not related with lower de novo synthesis but with its consumption.

The modulation of cholesterol metabolism evidenced in our study, could be due to the production of SCFAs as final products of bacterial activity in the digestive tract: SCFAs can decrease lipid levels in the blood by blocking the synthesis of hepatic cholesterol and transferring plasma cholesterol to the liver ${ }^{4,62}$.

This study clearly evidenced a novel role of L. rhamnosus on the modulation of the microbiota community in the zebrafish digestive tract. The digestive tract microbiome changes in response to the probiotic supplementation modulated the expression of a network of genes involved in the physiological control of lipogenesis, a concomitant reduction of total body cholesterol and triglycerides, an increase of non-polar fatty acids, an improvement of the intestinal epithelium structures (i.e. microvilli and enterocytes) and a reduction in enterocyte LDs, together with an increase in growth of probiotic treated zebrafish larvae.

The findings we report provide a novel gene network through which $L$. rhamnosus acts and, by inducing transcriptional changes, modulates lipid metabolism. Probiotic L. rhamnosus also i) modulates gut microbial composition, ii) increases FAs concentration, and iii) expands the absorptive surface of the intestinal epithelium by increasing microvilli and enterocytes heights. Since the zebrafish has become an established vertebrate model for a wide range of human diseases with particular strengths for biomedical research, the results discussed here suggest a possible use of L. rhamnosus for improving lipid metabolic disorder.

\section{Methods}

Animals and probiotic administration. Adult female and male zebrafish (D. rerio) were purchased from Acquario di Bologna (Italy) and acclimatized to the laboratory conditions $\left(27.0 \pm 0.5^{\circ} \mathrm{C}\right.$ under a $12: 12 \mathrm{~h}$ light: dark photoperiod). Pairs were spawned individually and larvae were raised under a $12: 12 \mathrm{~h}$ light: dark cycle at $27^{\circ} \mathrm{C}$. Embryos were collected and after hatching were divided into a control group and a probiotic-treated group. Larvae were fed a commercial diet (JBL flakes,

Germany) consisting of $43.00 \%$ crude protein, $8.30 \%$ crude fat, $8.10 \%$ ash, $1.9 \%$ fibre and $8.00 \%$ moisture content. The probiotic treatment consisted of the administration of L. rhamnosus IMC 501 ${ }^{\circledR}$ (C025396A; Synbiotec, Camerino, Italy) via the rearing water at a concentration of $10^{6}$ colony-forming units (CFU) according to previous studies $^{36,63-65}$. 

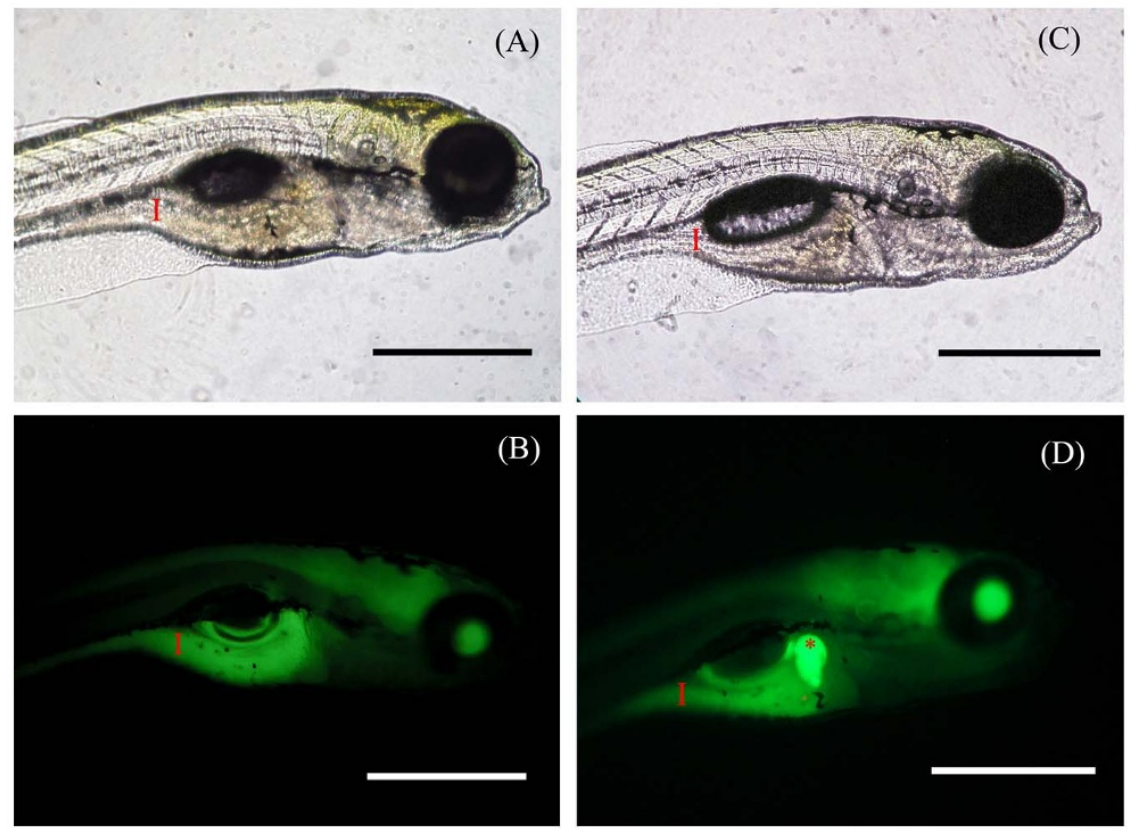

Figure 4 | BODIPY 505/515 staining evidenced an accumulation of non-polar fatty acids in probiotic treated gallbladder. Representative fluorescent images of live 6 dpf zebrafish soaked in BODIPY 505/515 for 1 hour. Images revealed green fluorescence in the intestine in both control (A-B) and probiotic treated groups (C-D). Probiotic treated larvae exhibited higher fluorescent signal in the gallbladder (D). Red asterisk: gallbladder; red arrow: intestine. Scale bar: $500 \mu \mathrm{m}$. (See also Figure S4).

The experiment was set up in triplicates, with three control tanks and three probiotic tanks and from each tank a pool of larvae was collected. The experiment was repeated three times.

Both control and probiotic groups were fed twice a day. At hatching, $96 \mathrm{hpf}, 6 \mathrm{dpf}$ and $8 \mathrm{dpf}$, larvae were euthanised using MS222 (100 $\left.\mathrm{mg} \mathrm{L}^{-1}\right)$ (Sigma-Aldrich) and stored at $-80^{\circ} \mathrm{C}$ for real time PCR analyses. At $96 \mathrm{hpf}, 6$ and $8 \mathrm{dpf}$ larvae were sampled for morphometric analyses. Since at $6 \mathrm{dpf}$, molecular analysis revealed significant changes of expression of all genes analysed, the high-throughput sequencing, HPLC, TEM and BODIPY staining were performed at this stage of development. Larvae from all stages, both control and the treated group were sampled $18 \mathrm{hrs}$ post meal, at $8 \mathrm{am}$. All procedures involving animals were conducted in accordance with the Italian law on animal experimentation and were approved by the Ethics Committee of Università Politecnica delle Marche (Prot \#63/INT/CESA12-16).
RNA extraction and cDNA synthesis. Total RNA was extracted from 15 whole larvae per tank using an RNAeasy ${ }^{\circledR}$ minikit (Qiagen, UK) according to Maradonna et al. ${ }^{66}$.

Real time PCR. PCRs were performed with the SYBR green method in an iQ5 iCycler thermal cycler (Bio-Rad laboratories). Triplicate PCRs were carried out for each sample analyzed. The reactions were set following Maradonna et al. ${ }^{66}$. The data obtained were analyzed using the iQ5 optical system software version 2.0 (Bio-Rad laboratories). Modification of gene expression is reported with respect to the control sample. The primer sequences for $a c t \beta, r p l p, m g l l, h n f 4 \alpha$, fit2, scap and $c c k$ were designed using Primer3 (210 v. 0.4.0), primers for agpat4 and dgat 2 were taken from Her et al. ${ }^{67}$.

The primer sequences are: agpat4 forward TGCTGAAAACTCAGTTGCTG, agpat4 reverse AGTAACCCAGTCTGCAGTTG, dgat2 forward
(A)

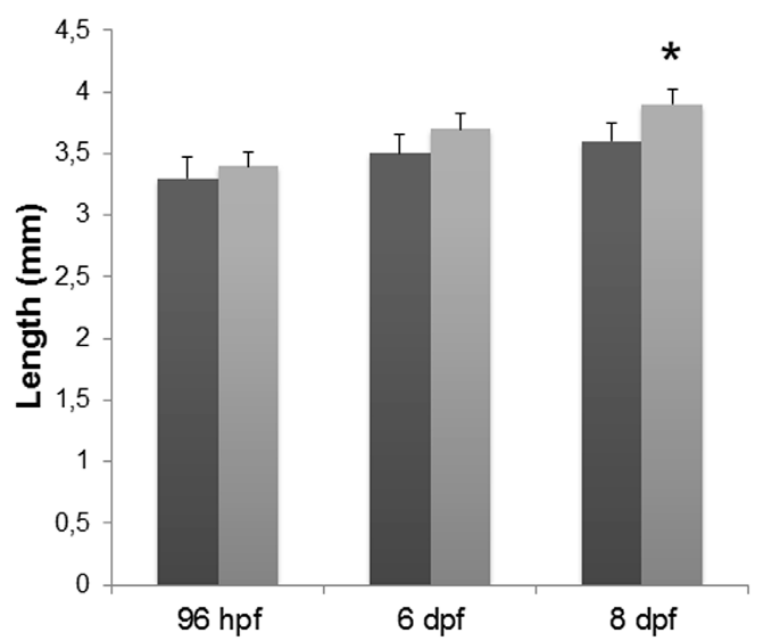

(B)

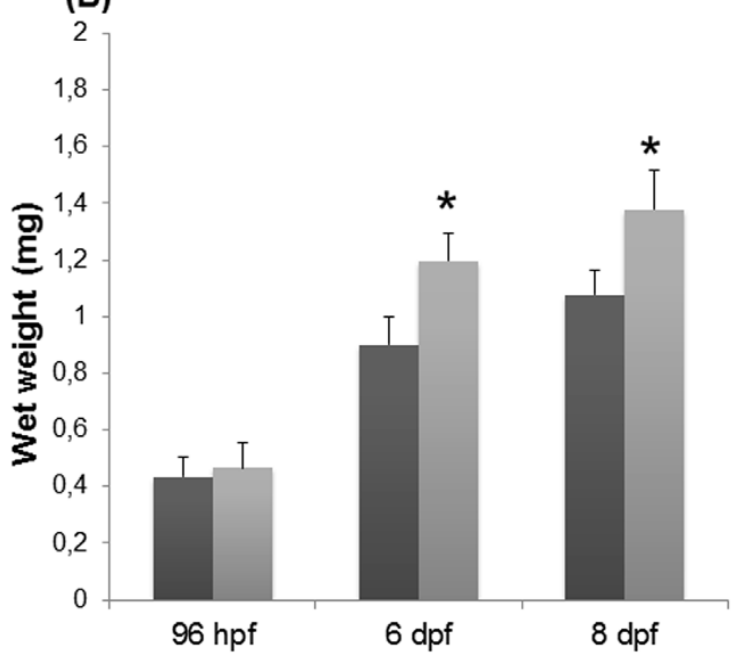

\section{Control Probiotic}

Figure $5 \mid$ L. rhamnosus administration increased larvae total length and body weight. Total length (A) and wet weight (B) of zebrafish larvae from control and probiotic groups collected at $96 \mathrm{hpf}, 6 \mathrm{dpf}$ and $8 \mathrm{dpf}$. Data are the mean \pm s.d. Asterisk indicates significant differences $(P<0.05)$. 
TTCCGGTGTCAAAAAGGGCT, dgat 2 reverse CAGCAGCAAAGAGCAAGCAA, $m g l l$ forward CGAGAGGCCGCAGGATTTTA, $m g l l$ reverse

TGAGTTTAGGAGCCAAGCG, $h n f 4 \alpha$ forward ACGGTTCGGCGAGCTGCTTC, reverse $h n f 4 \alpha$ TCCTGGACCAGATGGGGGTGT, fit 2 forward

CTGGTCTCCCTCCACAGCCGA, fit2 reverse ACACCAGCTGCCCTCCGCTT, scap forward GCTGTTACCCTCTGCTGAAG, scap reverse

TGAAACCGCTGCCTTGAC, $c c k$ forward CGCCTGCTGGACAAATCAAC, $c c k$ reverse TGCGGTATGAGCCTTTGGTT, rplp forward

CTGAACATCTCGCCCTTCTC- $3^{\prime}$, rplp reverse TAGCCGATCTGCAGACACAC, $\beta$-act forward GGTACCCATCTCCTGCTCCAA, $\beta$-act reverse

GAGCGTGGCTACTCCTTCACC.

\section{Digestive tract microbiome analysis}

DNA extraction and PCR. DNA was extracted from $100 \mathrm{mg}$ of the sterilized organisms, after a 30 min lysozyme incubation (50 mg $\mathrm{mL}^{-1}$ ) at $37^{\circ} \mathrm{C}$, using a QIAamp ${ }^{\circledR}$ Stool Mini Kit (Qiagen, Crawley, UK) according to the manufacturer's instructions, with some modifications in the cleanup and precipitation steps according to ${ }^{68}$. Briefly, after the removal of proteins, $460 \mu \mathrm{L}$ of extract product was added to an equal volume of ice-cold Tris-buffered phenol solution, left to stand on ice for $10 \mathrm{~min}$, then washed twice with an equal volume of chloroform, and centrifuged for $5 \mathrm{~min}$ at 3,000 g. The upper aqueous layer was isolated and the DNA precipitated overnight using an equal volume of ice-cold isopropanol and $10 \%$ sodium acetate, before centrifugation at $14,000 \mathrm{~g}$ for $30 \mathrm{~min}$ at $4^{\circ} \mathrm{C}$. The DNA pellet was washed twice using $300 \mu \mathrm{L}$ of $70 \%$ molecular biology-grade ethanol, before air drying for $5 \mathrm{~min}$, and resuspension overnight at $4^{\circ} \mathrm{C}$ in $30 \mu \mathrm{L}$ sterile water. The DNA concentration was determined at $260 \mathrm{~nm}$ using a NanoDropTM 1000 spectrophotometer (Thermo Scientific Ltd, DE, USA). All reagents used were molecular grade, purchased from Sigma-Aldrich (UK).

PCR amplification of the $16 \mathrm{~S}$ rRNA V3 region was conducted with a nested PCR strategy ${ }^{69}$, the external PCR was carried out using primers Eub8F (5'-AGAGTTTGATCMTGGCTCAG-' 3$)^{70}$ and $984 y$ R (5'-GTAAGGTTCYTCGCGT-3) ${ }^{71}$; the internal PCR was conducted using the primers P1F ( $5^{\prime}$-CCTACGGGAGGCAGCAG-3' + GC clamp) and P2R (5'-ATTACCGCGGCTGCTGG$\left.3^{\prime}\right)^{72}$. Two $\mu \mathrm{L}$ of DNA template was used in both reactions. The PCRs were performed in a Techne TC-312 cycler (Thermal Cyclers, Staffordshire, UK) for both reactions with 25 cycles of $95^{\circ} \mathrm{C}$ for $30 \mathrm{~s}, 50 / 53^{\circ} \mathrm{C}$ for $30 \mathrm{~s}, 72^{\circ} \mathrm{C}$ for $1 \mathrm{~min}$ and a final extension $72^{\circ} \mathrm{C}$ for $5 \mathrm{~min}$. The annealing temperatures were $50^{\circ} \mathrm{C}$ and $53^{\circ} \mathrm{C}$ for the external and internal PCRs, respectively.

High-throughput sequence analysis. PCR products were purified using a QIAquick PCR Purification Kit and quantified using a Qubit ${ }^{\circledR} 2.0$ Fluorometer (Invitrogen). Prior to sequencing the amplicons were assessed for fragment concentration using an Ion Library Quantitation Kit (LifeTechnologies ${ }^{\mathrm{TM}}$, USA), and concentrations were then adjusted to $26 \mathrm{pM}$. Amplicons were attached to Ion Sphere Particles (ISPs) using an Ion PGM ${ }^{\mathrm{TM}}$ Template OT2 200 kit (LifeTechnologies ${ }^{\mathrm{TM}}$, USA) according to the manufacturer's instructions. Multiplexed sequencing was conducted using a $316^{\mathrm{TM}}$ chip (LifeTechnologies ${ }^{\mathrm{TM}}$ ) on an Ion Torrent Personal Genome Machine (LifeTechnologies ${ }^{\mathrm{TM}}$ ). Sequences were binned by sample and filtered within the PGM software to remove low quality reads. Data were then exported as FastQ files.

Taxonomic analyses of sequence reads were performed after the removal of low quality scores (Q score $<20$ ) with FASTX-Toolkit (Hannon Lab, USA). Sequences were concatenated and sorted by sequence similarity into a single fasta file. Sequences were denoised and analyzed with QIIME ${ }^{73}$. Briefly, OTU mapping was performed using the USEARH quality filter pipeline ${ }^{20}$, to remove putatively erroneous reads (chimeras), then OTU picking was achieved with a minimum pairwise identity of $97 \%$. The most abundant sequence in each OTU were selected to assign a taxonomic classification based on the Greengenes database ${ }^{74}$ using the RDP classifier ${ }^{75}$, clustering the sequences at $97 \%$ similarity with a 0.80 confidence threshold.
PyNast was used to create a multiple alignment of the representative sequences for each OTU ${ }^{76}$ with minimum sequence length threshold of $120 \mathrm{bp}$ and $97 \%$ identification. Sequences were filtered to remove outliers and filter positions with gaps $(0.95)$ and singletons. Highest homologous species were identified at $97 \%$ and 120 bp using the resulting consensus sequences in CLC Bio Genomics Workbench v6.0.4 (QIAGEN, CLC Inc, Aarhus, Denmark) against the 16S microbial BLAST-NCBI database from 04/22/2013, after a de novo assembling with default parameters except that $80 \mathrm{bp}$ was used as the minimum contig length.

Alpha diversity metrics were calculated on rarefied OTU tables with QIIME to asses sampling depth coverage using observed species, phylogenetic diversity (PD), Chaol, Shannon's diversity index and Good's coverage. QIIME was also used to calculate Beta diversity metrics among samples using weighted Unifrac distances ${ }^{22}$ and BrayCurtis similarity ${ }^{23}$. The distance matrixes were represented by two dimensional principal coordinates analysis ( $\mathrm{PCoA})$ plots.

High-performance liquid chromatography (HPLC). The total lipid contents of $300 \mathrm{mg}$ of larvae at $6 \mathrm{dpf}$ were extracted with the chloroform-methanol method $(2: 1 \mathrm{v} / \mathrm{v})^{77}$. Cholesterol and TAG were quantified by HPLC-Light Scattering Detector (LSD) analyses using a LC-10AD VP gradient pump (Shimadzu, Kyoto, Japan), a VisionHT Silica $3 \mu \mathrm{m}$ column $(150 \times 2.1 \mathrm{~mm}$ i.d., Grace Davison Discovery Science, Deerfield, IL, USA) and a LSD (Sedex 55, S.E.D.E.RE., France), operating at $40^{\circ} \mathrm{C}$ and nitrogen pressure of $240 \mathrm{kPa}$. The chromatograms were acquired and the data handled using the Class-VP software (Shimadzu). A binary gradient system composed of hexane [eluent A] and 2-propanol [eluent B] was used following the solvent elution profile: $0 \mathrm{~min} 100 \% \mathrm{~A}, 10 \mathrm{~min} 90 \% \mathrm{~A}$, $15 \mathrm{~min} 80 \% \mathrm{~A}, 20-30 \mathrm{~min} 100 \% \mathrm{~A}$. The flow rate was $400 \mu \mathrm{L} \mathrm{min}{ }^{-1}$. Identification of cholesterol and TAG in the samples was carried out by comparison of their retention time with those of the respective commercial standards. Repeated injections of standard solutions were carried out to test the analytical precision. The relative standard deviations were less than 5\% for all the analyses, both considering the intradie precision, calculated on six repeated injections, and the interdie precision, evaluated over six days. Calibration curves, drawn for each lipid standard by injecting different concentrations $\left(5-40 \mu \mathrm{g} \mathrm{mL}{ }^{-1}\right.$ ), were used to quantify cholesterol, cholesteryl esters and TAG in the fish samples. All the HPLC analyses were carried out in triplicate. Cholesterol $(\geq 99 \%)$ and glyceryl trioleate $(\geq 99 \%)$ were purchased from Sigma-Aldrich (St. Louis, MO, USA). Hexane and 2-propanol were of highperformance liquid chromatography (HPLC) grade and were obtained from Merck (Darmstadt, Germany). The data obtained have been normalized to DNA content.

Transmission Electron Microscopy (TEM). Samples of 10 zebrafish larvae at $6 \mathrm{dpf}$ were fixed with $1 \%$ potassium dichromate, $1 \%$ osmium tetroxide and $2 \%$ glutaraldehyde in cacodylate buffer $(0.1 \mathrm{M}, \mathrm{pH} 7.2)$ for 5 hours at $4^{\circ} \mathrm{C}$. Samples were washed four times with cacodylate buffer for 12 hours at $4^{\circ} \mathrm{C}$, then dehydrated with a graded acetone series (from $50 \%$ to $100 \%$ ) and embedded in epon-based resin. All reagents were purchased from AGAR Scientific (UK). The thin $(1 \mu \mathrm{m})$ and ultrathin sections $(60-80 \mathrm{~nm})$ were obtained with a Reichert Ultracut ultramicrotome using a diamond knife and were collected on glass slides or copper grids. The thin sections were stained with $1 \%$ toluidine blue and images were captured using a computer-assisted image analysis system which includes a Zeiss microscope equipped with a colour video camera (Axio Cam MRC, Arese Milano Italy). The ultrathin sections were stained with $1 \%$ uranyl acetate and Reynolds lead citrate and then observed by TEM (JEOL 1200 EXII, JEOL Tokyo, Japan). Micrographs were acquired with an Olympus SIS VELETA CCD camera equipped with the iTEM software. Microvilli lengths, enterocyte lengths and lipid droplet diameters were determined 
from 10 fish per group; 6 images were taken for each intestine and 12 microvilli and enterocytes were measured per image. Enterocyte lengths were measured starting from their basal membrane connected with lamina propria up to the base of microvilli, while microvilli were measured starting from their base to the apical tip. All measurements were taken from micrographs using Software Image $\mathrm{J}^{78}$. In all cases, measurements were made using sections in which enterocytes were cut in their entire length, with the nuclei visible and aligned, to ensure that the section plane was appropriate.

BODIPY 505/515 staining. Fifteen larvae at $6 \mathrm{dpf}$ were placed into 12 -well plastic dishes (3 larvae per well) and soaked in $100 \mu \mathrm{M}$ BODIPY 505/515 (4,4-Difluoro-1,3,5,7-Tetramethyl-4-Bora-3a,4aDiaza-s-Indacene) (Invitrogen) diluted in $2 \%$ DMSO, then incubated for 1 hour at $28^{\circ} \mathrm{C}$ in the dark. Larvae were anaesthetized using MS222 (100 $\left.\mathrm{mg} \mathrm{L}^{-1}\right)$ (Sigma- Aldrich, Milan, Italy) and fluorescent images were obtained by a Bio-Rad MRC 1024 confocal microscope (Bio-Rad Microscience Ltd, Bio-Rad House, Hemel Hempstead, UK); equipped with a $10 \times$ objective. Bodipy 505/515 was excited at $505 \mathrm{~nm}$ (blue light) and emitted a spectrum of wavelength light which peaked at $515 \mathrm{~nm}$. Regions of Interest (ROI) of 15 galbladders and 15 intestines per group were selected. Fluorescence intensity (arbitrary units a.u.) was determined using Image J software.

Morphological analysis. At each time point larval length was measured using a Stemi 2000 micrometric Microscope (Zeiss Vision Italia, Castiglione Orona, Italy) and weight was determined using a Microbalance (OHAUS Explorer E11140, Pine Brook, NJ, USA).

Statistical analysis. Results were expressed as the mean \pm s.d. Statistical differences were determined using ANOVA, followed by Bonferroni's multiple comparison test. All statistical analyses were performed using Prism 6 (GraphPad Software, San Diego, CA, USA). A t-test was used to identify significant differences in relative abundance of bacterial taxa. P-values $<0.05$ were considered significant.

1. Semova, I. et al. Microbiota regulate intestinal absorption and metabolism of fatty acids in the zebrafish. Cell Host \& Microbe 12, 277-288 (2012).

2. Bäckhed, F., Manchester, J. K., Semenkovich, C. F. \& Gordon, J. I. Mechanisms underlying the resistance to diet-induced obesity in germ-free mice. Proceedings of the National Academy of Sciences 104, 979-984 (2007).

3. Cani, P. D. \& Delzenne, N. M. The role of the gut microbiota in energy metabolism and metabolic disease. Curr. Pharm. Des. 15, 1546-1558 (2009).

4. St-Onge, M.-P., Farnworth, E. R. \& Jones, P. J. Consumption of fermented and nonfermented dairy products: effects on cholesterol concentrations and metabolism. The American journal of clinical nutrition 71, 674-681 (2000).

5. Ooi, L.-G. \& Liong, M.-T. Cholesterol-lowering effects of probiotics and prebiotics: a review of in vivo and in vitro findings. International journal of molecular sciences 11, 2499-2522 (2010).

6. Buhman, K. F., Accad, M. \& Farese, R. V. Mammalian acyl-CoA: cholesterol acyltransferases. Biochimica et Biophysica Acta (BBA)-Molecular and Cell Biology of Lipids 1529, 142-154 (2000).

7. Coleman, R. A. \& Lee, D. P. Enzymes of triacylglycerol synthesis and their regulation. Prog. Lipid Res. 43, 134-176 (2004).

8. Yen, C.-L. E., Stone, S. J., Koliwad, S., Harris, C. \& Farese, R. V. Thematic review series: glycerolipids. DGAT enzymes and triacylglycerol biosynthesis. J. Lipid Res. 49, 2283-2301 (2008).

9. Gonzalez-Baró, M. R., Lewin, T. M. \& Coleman, R. A. Regulation of Triglyceride Metabolism II. Function of mitochondrial GPAT1 in the regulation of triacylglycerol biosynthesis and insulin action. American Journal of PhysiologyGastrointestinal and Liver Physiology 292, G1195-G1199 (2007).

10. Gimeno, R. E. \& Cao, J. Thematic review series: glycerolipids. Mammalian glycerol-3-phosphate acyltransferases: new genes for an old activity. J. Lipid Res. 49, 2079-2088 (2008).

11. Zimmermann, R. et al. Fat mobilization in adipose tissue is promoted by adipose triglyceride lipase. Science 306, 1383-1386 (2004).

12. Chang, J. et al. Expression of proteins associated with adipocyte lipolysis was significantly changed in the adipose tissues of the obese spontaneously hypertensive/NDmcr-cp rat. Diabetology \& metabolic syndrome 6, 8 (2014).
13. Langin, D. Adipose tissue lipolysis as a metabolic pathway to define pharmacological strategies against obesity and the metabolic syndrome. Pharmacol. Res. 53, 482-491 (2006).

14. Ho, S.-Y., Delgado, L. \& Storch, J. Monoacylglycerol Metabolism in Human Intestinal Caco-2 Cells evidence for metabolic compartmentation and hydrolysis. J. Biol. Chem. 277, 1816-1823 (2002).

15. Fredrikson, G., Tornqvist, H. \& Belfrage, P. Hormone-sensitive lipase and monoacylglycerol lipase are both required for complete degradation of adipocyte triacylglycerol. Biochimica et Biophysica Acta (BBA)-Lipids and Lipid Metabolism 876, 288-293 (1986).

16. Long, J. Z. \& Cravatt, B. F. The metabolic serine hydrolases and their functions in mammalian physiology and disease. Chem. Rev. 111, 6022-6063 (2011).

17. Shi, Y. \& Cheng, D. Beyond triglyceride synthesis: the dynamic functional roles of MGAT and DGAT enzymes in energy metabolism. American Journal of Physiology-Endocrinology And Metabolism 297, E10-E18 (2009).

18. Buhman, K. K. et al. DGAT1 is not essential for intestinal triacylglycerol absorption or chylomicron synthesis. J. Biol. Chem. 277, 25474-25479 (2002).

19. Gross, D. A., Zhan, C. \& Silver, D. L. Direct binding of triglyceride to fat storageinducing transmembrane proteins 1 and 2 is important for lipid droplet formation. Proceedings of the National Academy of Sciences 108, 19581-19586 (2011).

20. Edgar, R. C. Search and clustering orders of magnitude faster than BLAST. Bioinformatics 26, 2460-2461 (2010).

21. Faith, D. P. Conservation evaluation and phylogenetic diversity. Biol. Conserv. 61, 1-10 (1992).

22. Lozupone, C. A., Hamady, M., Kelley, S. T. \& Knight, R. Quantitative and qualitative $\beta$ diversity measures lead to different insights into factors that structure microbial communities. Appl. Environ. Microbiol. 73, 1576-1585 (2007).

23. Bray, J. R. \& Curtis, J. T. An ordination of the upland forest communities of southern Wisconsin. Ecol. Monogr. 27, 325-349 (1957).

24. Takeuchi, K. \& Reue, K. Biochemistry, physiology, and genetics of GPAT, AGPAT, and lipin enzymes in triglyceride synthesis. American Journal of Physiology-Endocrinology And Metabolism 296, E1195-E1209 (2009).

25. Hayhurst, G. P., Lee, Y.-H., Lambert, G., Ward, J. M. \& Gonzalez, F. J. Hepatocyte nuclear factor $4 \alpha$ (nuclear receptor $2 \mathrm{~A} 1$ ) is essential for maintenance of hepatic gene expression and lipid homeostasis. Mol. Cell. Biol. 21, 1393-1403 (2001).

26. Brown, M. S. \& Goldstein, J. L. The SREBP pathway: regulation of cholesterol metabolism by proteolysis of a membrane-bound transcription factor. Cell $\mathbf{8 9}$, 331-340 (1997)

27. Little, T. J., Horowitz, M. \& Feinle-Bisset, C. Role of cholecystokinin in appetite control and body weight regulation. Obesity reviews 6, 297-306 (2005).

28. Ahmadian, M., E Duncan, R., Jaworski, K., Sarkadi-Nagy, E. \& Sul, H. S. Triacylglycerol metabolism in adipose tissue. Future Lipidol. 2, 229-237 (2007)

29. Levy, E. et al. Intestinal cholesterol transport proteins: an update and beyond. Curr. Opin. Lipidol. 18, 310-318 (2007).

30. Walley, A. J., Blakemore, A. I. \& Froguel, P. Genetics of obesity and the prediction of risk for health. Hum. Mol. Genet. 15, R124-R130 (2006).

31. Anderson, J. L., Carten, J. D. \& Farber, S. A. Zebrafish lipid metabolism: from mediating early patterning to the metabolism of dietary fat and cholesterol. Methods Cell Biol. 101, 111 (2011).

32. Wallace, K. N. \& Pack, M. Unique and conserved aspects of gut development in zebrafish. Dev. Biol. 255, 12-29 (2003).

33. Carten, J. D., Bradford, M. K. \& Farber, S. A. Visualizing digestive organ morphology and function using differential fatty acid metabolism in live zebrafish. Dev. Biol. 360, 276-285 (2011).

34. Iqbal, J. \& Hussain, M. M. Intestinal lipid absorption. American Journal of Physiology-Endocrinology And Metabolism 296, E1183-E1194 (2009).

35. Tanaka, T. et al. Free fatty acids induce cholecystokinin secretion through GPR120. Naunyn-Schmiedeberg's Arch. Pharmacol. 377, 523-527 (2008).

36. Gioacchini, G. et al. Probiotics can induce follicle maturational competence: the Danio rerio case. Biol. Reprod. 86, 65 (2012).

37. Sieuwerts, S. et al. Mixed-culture transcriptome analysis reveals the molecular basis of mixed-culture growth in Streptococcus thermophilus and Lactobacillus bulgaricus. Appl. Environ. Microbiol. 76, 7775-7784 (2010).

38. Fukushima, M. \& Nakano, M. Effects of a mixture of organisms, Lactobacillus acidophilus or Streptococcus faecalis on cholesterol metabolism in rats fed on a fat-and cholesterol-enriched diet. Br. J. Nutr. 76, 857-867 (1996).

39. Dimitroglou, A. et al. Microbial manipulations to improve fish health and production-a Mediterranean perspective. Fish Shellfish Immunol. 30, 1-16 (2011).

40. Donohoe, D. R. et al. The microbiome and butyrate regulate energy metabolism and autophagy in the mammalian colon. Cell metabolism 13, 517-526 (2011)

41. Resta, S. C. Effects of probiotics and commensals on intestinal epithelial physiology: implications for nutrient handling. The Journal of physiology 587, 4169-4174 (2009).

42. Cashman, K. Prebiotics and calcium bioavailability. Curr. Issues Intestinal Microbiol. 4, 21-32 (2003).

43. Hijova, E. \& Chmelarova, A. Short chain fatty acids and colonic health. Bratisl. Lek. Listy 108, 354 (2007) 
44. Scholz-Ahrens, K. E. et al. Prebiotics, probiotics, and synbiotics affect mineral absorption, bone mineral content, and bone structure. The Journal of nutrition 137, 838S-846S (2007).

45. Yamaguchi, K. et al. Inhibiting triglyceride synthesis improves hepatic steatosis but exacerbates liver damage and fibrosis in obese mice with nonalcoholic steatohepatitis. Hepatology 45, 1366-1374 (2007).

46. Agarwal, A. K. \& Garg, A. Congenital generalized lipodystrophy: significance of triglyceride biosynthetic pathways. Trends in Endocrinology \& Metabolism 14, 214-221 (2003).

47. Turkish, A. \& Sturley, S. L. Regulation of triglyceride metabolism. I. Eukaryotic neutral lipid synthesis:"Many ways to skin ACAT or a DGAT". American Journal of Physiology-Gastrointestinal and Liver Physiology 292, G953-G957 (2007).

48. Farese Jr, R. V. \& Walther, T. C. Lipid droplets finally get a little RESPECT. Cell 139, 855-860 (2009).

49. Kadereit, B. et al. Evolutionarily conserved gene family important for fat storage. Proceedings of the National Academy of Sciences 105, 94-99 (2008).

50. Guo, Y. et al. Functional genomic screen reveals genes involved in lipid-droplet formation and utilization. Nature 453, 657-661 (2008).

51. Sáenz de Rodrigáñez, M. et al. Effect of dietary administration of probiotics on growth and intestine functionality of juvenile Senegalese sole (Solea senegalensis, Kaup 1858). Aquacult. Nutr. 15, 177-185 (2009).

52. Hofmann, A. \& Hagey, L. Bile acids: chemistry, pathochemistry, biology, pathobiology, and therapeutics. Cell. Mol. Life Sci. 65, 2461-2483 (2008).

53. Gérard, P. Metabolism of Cholesterol and Bile Acids by the Gut Microbiota Pathogens 3, 14-24 (2013).

54. Pavlović, N., Stankov, K. \& Mikov, M. Probiotics-interactions with bile acids and impact on cholesterol metabolism. Appl. Biochem. Biotechnol. 168, 1880-1895 (2012).

55. Dawes, L. G., Muldoon, J. P., Greiner, M. A. \& Bertolotti, M. Cholecystokinin increases bile acid synthesis with total parenteral nutrition but does not prevent stone formation. J. Surg. Res. 67, 84-89 (1997).

56. Sitzmann, J., Pitt, H., Steinborn, P., Pasha, Z. \& Sanders, R. Cholecystokinin prevents parenteral nutrition induced biliary sludge in humans. Surg. Gynecol. Obstet. 170, 25-31 (1990).

57. Long, J. Z., Nomura, D. K. \& Cravatt, B. F. Characterization of monoacylglycerol lipase inhibition reveals differences in central and peripheral endocannabinoid metabolism. Chem. Biol. 16, 744-753, doi:http://dx.doi.org/10.1016/j.chembiol. 2009.05.009 (2009).

58. Schroeder, F. et al. Role of fatty acid binding proteins and long chain fatty acids in modulating nuclear receptors and gene transcription. Lipids 43, 1-17 (2008).

59. Iwayanagi, Y., Takada, T. \& Suzuki, H. HNF4 $\alpha$ is a crucial modulator of the cholesterol-dependent regulation of NPC1L1. Pharm. Res. 25, 1134-1141 (2008).

60. Kumar, M. et al. Cholesterol-lowering probiotics as potential biotherapeutics for metabolic diseases. Experimental diabetes research 2012, (2012).

61. Espenshade, P. J. SREBPs: sterol-regulated transcription factors. J. Cell Sci. 119, 973-976 (2006).

62. Bisanz, J. E. \& Reid, G. Unraveling how probiotic yogurt works. Science translational medicine 3, 106ps141-106ps141 (2011).

63. Sieuwerts, S., De Bok, F., Mols, E., De Vos, W. \& van Hylckama Vlieg, J. A simple and fast method for determining colony forming units. Lett. Appl. Microbiol. 47, 275-278 (2008).

64. Gioacchini, G. et al. Interplay between autophagy and apoptosis in the development of Danio rerio follicles and the effects of a probiotic. Reprod. Fertil. Dev. 25, 1115-1125 (2013).

65. Gatesoupe, F. The use of probiotics in aquaculture. Aquaculture 180, 147-165 (1999).

66. Maradonna, F. et al. Probiotic supplementation promotes calcification in Danio rerio larvae: a molecular study. PloS one $\mathbf{8}$, e83155 (2013).

67. Her, G. M. et al. Overexpression of gankyrin induces liver steatosis in zebrafish Danio rerio. Biochimica et Biophysica Acta (BBA)-Molecular and Cell Biology of Lipids 1811, 536-548 (2011).
68. Waines, P. L., Moate, R., Moody, A. J., Allen, M. \& Bradley, G. The effect of material choice on biofilm formation in a model warm water distribution system. Biofouling 27, 1161-1174 (2011).

69. Bakke, I., De Schryver, P., Boon, N. \& Vadstein, O. PCR-based community structure studies of Bacteria associated with eukaryotic organisms: A simple PCR strategy to avoid co-amplification of eukaryotic DNA. J. Microbiol. Methods $\mathbf{8 4}$ 349-351 (2011).

70. Weisburg, W. G., Barns, S. M., Pelletier, D. A. \& Lane, D. J. 16S ribosomal DNA amplification for phylogenetic study. J. Bacteriol. 173, 697-703 (1991).

71. Wang, Y. \& Qian, P.-Y. Conservative fragments in bacterial 16S rRNA genes and primer design for $16 \mathrm{~S}$ ribosomal DNA amplicons in metagenomic studies. PloS one 4, e7401 (2009).

72. Muyzer, G., De Waal, E. C. \& Uitterlinden, A. G. Profiling of complex microbial populations by denaturing gradient gel electrophoresis analysis of polymerase chain reaction-amplified genes coding for $16 \mathrm{~S}$ rRNA. Appl. Environ. Microbiol. 59, 695-700 (1993).

73. Caporaso, J. G. et al. QIIME allows analysis of high-throughput community sequencing data. Nat. Methods 7, 335-336 (2010).

74. DeSantis, T. Z. et al. Greengenes, a chimera-checked $16 \mathrm{~S}$ rRNA gene database and workbench compatible with ARB. Appl. Environ. Microbiol. 72, 5069-5072 (2006).

75. Wang, Q., Garrity, G. M., Tiedje, J. M. \& Cole, J. R. Naive Bayesian classifier for rapid assignment of rRNA sequences into the new bacterial taxonomy. Appl. Environ. Microbiol. 73, 5261-5267 (2007).

76. Caporaso, J. G. et al. PyNAST: a flexible tool for aligning sequences to a template alignment. Bioinformatics 26, 266-267 (2010).

77. Folch, J., Lees, M. \& Sloane-Stanley, G. A simple method for the isolation and purification of total lipids from animal tissues. J. Biol. Chem. 226, 497-509 (1957).

78. Abràmoff, M. D., Magalhães, P. J. \& Ram, S. J. Image processing with ImageJ. Biophotonics international 11, 36-42 (2004).

\section{Acknowledgments}

The authors wish to thank Dr Michele Kiernan and Tony Reynolds from the Systems Biology Centre (Plymouth University) for conducting the Ion Torrent PGM analysis. The study was supported by FA 2013, PRIN 2010-2011 prot 2010W87LBJ, EU COST AQUAGAMETS 2012 FA12025 and the Seale-Hayne Educational Trust.

\section{Author contributions}

Conceived and designed the experiments: S.F. and O.C. Performed the experiments: S.F., F.M., A.R.T., G.G., A.R., S.P., L.C. and I.O. Analyzed the data: S.F., S.P., A.R., D.M. and O.C Contributed reagents/materials/analysis tools: S.P., D.M., A.R. and O.C. Wrote the paper: S.F., S.P., D.M., A.R. and O.C.

\section{Additional information}

Supplementary information accompanies this paper at http://www.nature.com/ scientificreports

Competing financial interests: The authors declare no competing financial interests. How to cite this article: Falcinelli, S. et al. Lactobacillus rhamnosus lowers lipid content by changing gut microbiota and host transcription of genes involved in lipid metabolism in zebrafish. Sci. Rep. 5, 9336; DOI:10.1038/srep09336 (2015).

This work is licensed under a Creative Commons Attribution 4.0 International License. The images or other third party material in this article are included in the article's Creative Commons license, unless indicated otherwise in the credit line; if the material is not included under the Creative Commons license, users will need to obtain permission from the license holder in order to reproduce the material. To view a copy of this license, visit http://creativecommons.org/licenses/by/4.0/ 